\title{
Modelling of Electric Fields inside Spacecraft Dielectrics using In-Orbit Charging Current Data
}

\author{
Keith A. Ryden, Member, IEEE, Alex D. P. Hands, Member, IEEE
}

\begin{abstract}
Internal charging caused by energetic electrons is a recognized threat to critical space infrastructure such as navigation and communication satellites. In this paper the electric field developed inside selected on-board dielectrics over a 10-year period in a GPS-like orbit is modelled using actual charging currents measured directly in orbit. The charging currents provide both charge deposition and dose rate inputs to the model, the latter allowing the introduction of radiation induced conductivity (RIC) to improve realism. As expected we find that RIC is a mitigating factor for the electric fields but they can still become very large e.g. a $1.0 \mathrm{~mm}$ thickness of PEEK under $0.5 \mathrm{~mm}$ of Al shielding would be at risk of breakdown almost throughout the mission. We also find that RIC tends to reduce sensitivity to space weather perturbations of the environment such as the April 2010 storm event. This seems physically reasonable but we also know that some satellite anomalies do correlate quite well with space weather and short term (daily) electron fluence increases. We recommend that correlation of anomaly data sets with electric field models of this type is undertaken in future: this will require accurate materials parameters and also needs to take account of sudden depletion of the electric field due to discharges. In addition more charging current sensors with greater shielding levels $(>2 \mathrm{~mm}$ Al equivalent) should be flown to allow modeling of a wider range of realistic cases, including inside well-shielded electronic boxes.
\end{abstract}

Index Terms-internal charging, electric fields, space weather, medium Earth orbit.

\section{INTRODUCTION}

$I^{2}$ NTERNAL charging [1], [2] is a recognized space weather risk to critical space infrastructure such as navigation and communication satellites and has been responsible for some of the most significant anomalies and failures experienced over the space age. For example the sudden disabling of three geostationary spacecraft (ANIK E1, E2 and Intelsat K) on the same day in January 1994 [3] was attributed to charging inside

Keith Ryden and Alex Hands are with the Surrey Space Centre, University of Surrey, Guildford, GU2 7XH, UK (e-mail: k.ryden@surrey.ac.uk)

We would like to acknowledge funding support for this work from the European Union (EU) Spacestorm project (Grant no. 606716) and from the European Space Agency (ESA) for this work. relatively lightly shielded equipment boxes after a period of elevated high-energy electron fluxes. Much longer data sets [4] from the 1990s showed conclusively that internal charging was an effective cause of satellite anomalies which has been backed up by numerous other studies. Wrenn [4] used correlations between anomalies and the daily (external) fluences of $>2 \mathrm{MeV}$ electrons to define a number of thresholds for signaling the likelihood of internal charging problems. Today most geostationary satellite operators use the $>2 \mathrm{MeV}$ (external) fluxes measured by the GOES spacecraft (available from SWPC [5]) as a key space weather risk indicator: SWPC and other space weather bodies send out alerts to operators based on both a $>2 \mathrm{MeV}$ flux level and a threshold daily fluence. However it has always been apparent that in even in the most convincing anomaly vs flux (or fluence) correlations there is never a perfect correspondence e.g. anomalies can occur when fluxes are low and vice versa.

Of course the electron fluxes/fluences are an input into the internal charging problem rather than the output: the final output is whether an electrostatic discharge (ESD) occurs which can lead to an anomaly. Penetrating electron fluxes create internal charging currents which in turn cause build-up of charge and thus of electric fields. Electric field is the most critical parameter, since when it exceeds the dielectric strength a breakdown will occur.

Satellite designers tend to focus on assessing and limiting either the internal charging current density or the electric field within sensitive dielectrics (rather than dealing in electron fluxes within the spacecraft) and safety thresholds are recommended in the main spacecraft charging standards [6], [7]. In most cases shielding is used to ensure the relevant criteria are met although other approaches such as minimizing dielectric thickness may also be practical. The relevant 'worst case' external environments are usually defined from one of a number of engineering models including SOPA [6] (geostationary only), FLUMIC [8][9] and MOBE-DIC [10]. These models tend to be based on short term peaks (typically averaged over 1 day).

For the current-limit design method a maximum mean (internal) charging current of $0.1 \mathrm{pAcm}^{-2}$ over 10 or 24 hours is a widely applied criterion which was derived from the results of the CRRES IDM flight experiment [11], [12] in the early 
1990s. A lower (internal) current threshold of $0.02 \mathrm{pAcm}^{-2}$ is also given in [7] for low temperature situations. The advantage of the current-limit method is simplicity since there is no need for detailed knowledge of dielectric material conductivity parameters because charge build-up is not part of the evaluation. Such charging current densities have also been measured on-board certain satellites with low-cost instruments such as SURF [13].

In order to perform a design based on electric field criteria, spacecraft engineers can use ESA's DICTAT or NASA's NUMIT threat assessment tools which can calculate maximum fields for 'worst case' conditions. A safe upper electric field of $10^{7} \mathrm{Vm}^{-1}$ is usually adopted but a safety margin of up to a factor 10 may also be applied to this number. An assessment based on electric field should ultimately be more accurate as it takes account of dielectric charge storage/leakage properties but this assumes that suitable materials data is available as an input. In reality it is often a challenge to obtain good materials data with the necessary provenance.

If the electric field developed within a dielectric in-orbit could be known this would be a very valuable risk indicator. However such measurements are difficult and have not been achieved in a flight experiment to date. As a stepping stone towards this goal however, Bodeau [14] has recently modeled the charge density (which is directly proportional to electric field) accumulated in a dielectric in orbit using actual space weather external flux measurements as the primary input. Bodeau used GOES $>2 \mathrm{MeV}$ daily electron fluences over a period of $>20$ years, fitted a fixed spectrum (AE8 adjusted in amplitude according to the daily fluence), transported the resulting spectrum through a given amount of shielding and then modelled the internal electric field build-up using a capacitor model. In so doing he found that the $0.1 \mathrm{pA} \mathrm{cm}$ internal current density threshold may be inadequate for dielectrics with extremely low conductivities where such average currents persist over weeks or months. Such modeling is highly beneficial but there are limitations to what has been done, notably: i) the use of a fixed electron spectrum since in practice the electron spectrum is changing constantly and ii) the absence of any radiation induced conductivity (RIC) which is a significant mitigating factor in many dielectrics.

The fixed spectrum problem could be overcome by fitting variable spectra to the GOES two-channel data but this could lead to large errors. Mulligan Skov et al [15] have modelled charge accumulation in materials in geostationary transfer orbit (Van Allen Probes) using high resolution electron spectra from the MagEIS instrument (10 channels). In this paper we circumvent the variable spectrum problem by using charging current measurements obtained in flight by the SURF instrument rather than flux. This type of measurement naturally takes account of the changing electron spectrum.

Ignoring RIC is a conservative and thus safe approximation especially, as [14] points out, if the charge is trapped in a thick electrically isolated conductor on top of the dielectric (e.g., ungrounded spot shields, thermal heat sinks), or in the upper layers of a very thick dielectric. RIC may then be negligible in the underlying dielectric, so we end up with a two-layer model dielectric approximation to study bulk charging. However RIC is a genuine mitigating factor and including it in the modelling should lead to improved accuracy. To achieve this goal, reliable dielectric RIC parameters need to be available as well as the dose rate for the appropriate dielectric leakage path (dose rate varies depending on the space weather conditions). We use currents measured by the SURF instrument to calculate dose rates (as well as charge deposition rates) which can then be combined with RIC parameters to calculate electric fields.

\section{SURF INTERNAL CHARGING SENSOR}

Our internal charging current measurements are from the 'SURF' sensor which is described at length in [16]. A sensor of this type has been flying now in medium Earth orbit for over ten years [17] on the Giove-A spacecraft: this vehicle is located in a GPS-type medium Earth orbit of altitude $23,300 \mathrm{~km}$ and inclination $56^{\circ}$. SURF on Giove-A measures deposited currents in three stacked collector plates as illustrated in Figure 1. The plate thicknesses and shields are chosen to be representative of many structures and shielding levels in a spacecraft, although it should be realized that many items will be afforded greater levels of shielding to meet either existing charging guidelines [6], [7] or total dose protection requirements. Data is available at 5 minute resolution. Daily average current densities (which we shorten to 'currents') in the top plate are plotted in Figure 2. Note the sharp peak in daily average currents in April 2010 which remain the highest currents observed at the time of writing. But as we see later April 2010 was not necessarily the peak risk period for ESD events. Also note that there is a significant data gap in early 2013 so results from this period and its immediate aftermath must be treated with caution; there are also a few other occasional data drop outs.

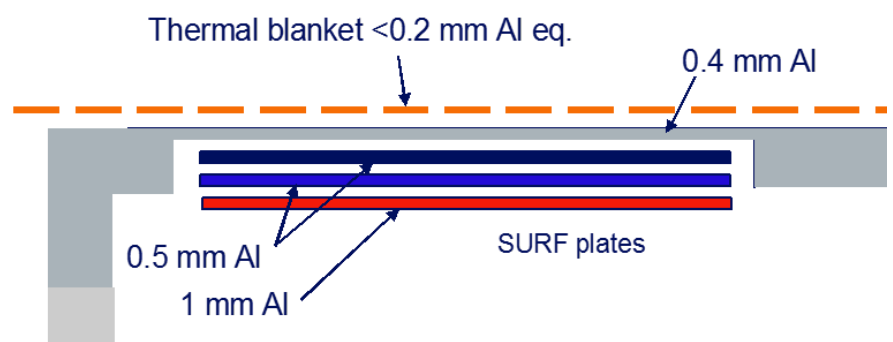

Figure 1 Illustration of the collector plate arrangements for the SURF sensor on Giove-A. Total shielding above the top plate is $\sim 0.5 \mathrm{~mm} \mathrm{Al}$ equivalent. For our 1-D capacitor charging model the top plate represents the absorber, so the current measured is the charging input.

\section{INITIAL ONE-DIMENSIONAL MODEL FOR DIELECTRIC CHARGING}

Following [14] we use a simple 1-D capacitor model to enable a simple calculation of the electric field inside a virtual 


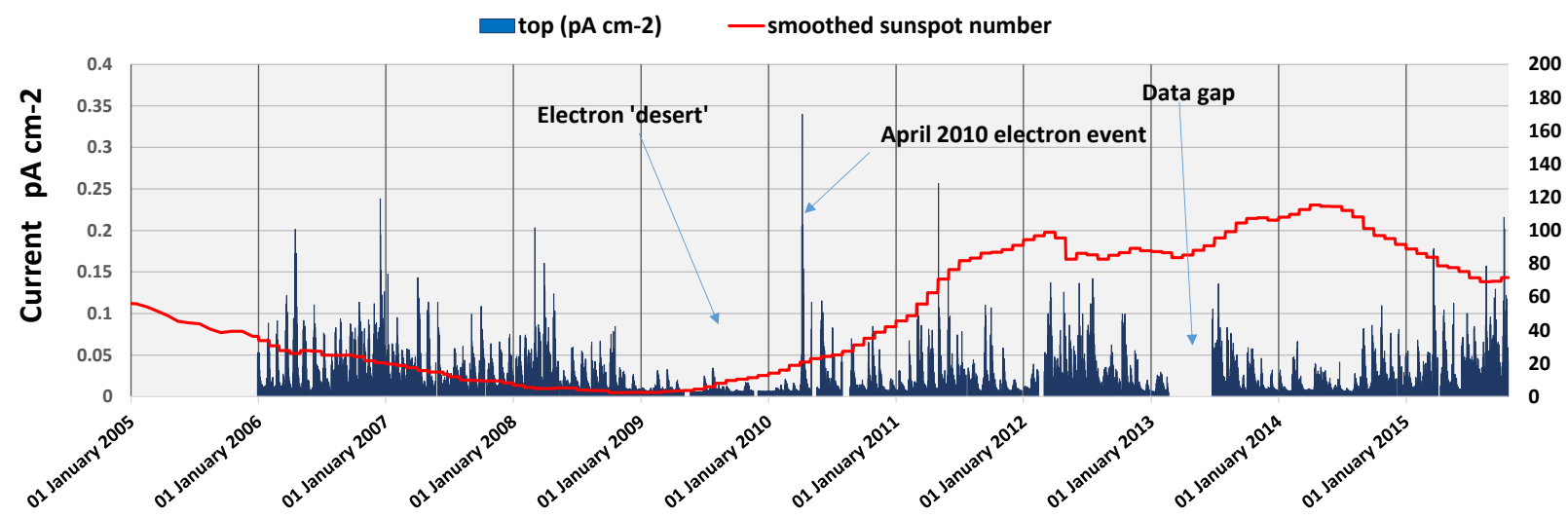

Figure 2 Top plate current (daily average) over the course of the Giove-A mission. Also plotted is the smoothed sunspot number (red line). These currents are used as the input to the simple 1-D charging model.

dielectric i.e. there is a top conductor of the capacitor into which current is injected which represents the total current deposited throughout the associated virtual dielectric. The current we inject in this model is that collected in the top plate of the SURF instrument. An electric field is then calculated across the virtual dielectric, the thickness of which is such that its areal density matches the areal density of the collector (i.e. the top SURF plate, $0.5 \mathrm{~mm}$ aluminum equivalent (Al-eq)). As most dielectrics are less dense than $\mathrm{Al}\left(2.7 \mathrm{~g} \mathrm{~cm}^{-3}\right)$ the virtual dielectric thickness is proportionately greater as illustrated in Table 1 (which is also used in the electric field calculation).

TABLE 1

THICKNESSES OF VARIOUS DIELECTRICS EQUIVALENT TO 0.5MM AL. (I.E. SAME CURRENT COLLECTION CAPABILITY)

\begin{tabular}{|l|l|l|}
\hline Material & $\begin{array}{l}\text { Density } \\
\left(\mathrm{g} \mathrm{cm}^{-3}\right)\end{array}$ & $\begin{array}{l}\text { Thickness } \\
\text { equivalent to } \\
\text { Al }\end{array}$ \\
\hline PEEK & $1.3 \mathrm{~mm})$ \\
\hline Kapton & 1.42 & 1.04 \\
\hline Teflon FEP & 2.15 & 0.95 \\
\hline
\end{tabular}

We assume the bottom surface of the (imaginary) dielectric (bottom plate of the capacitor) is grounded and thus charge can leak out there depending on the conductivity of the material. In our initial modelling leakage rates depend upon the dark conductivity only. Dark conductivity, $\sigma_{0}$, is usually dependent upon temperature but for this model we assume room temperature conditions. Dark conductivity at room temperature for materials of interest for internal charging usually lies in the region from $1 \times 10^{-16} \Omega^{-1} \mathrm{~m}^{-1}$ to $1 \times 10^{-18} \Omega^{-1}$ $\mathrm{m}^{-1}$. Assuming a relative permittivity of 2 (typical value), the corresponding range of charging time constants range from about 2 to 200 days.

We then calculate the electric field (E) each day by taking account of i) the charging curve (the rise towards a 'final' value) and ii) the decay curve (the natural decay due to leakage) i.e.:

$$
E(t+\Delta t)=E(t) \cdot e^{-\left(\frac{\Delta t}{\tau}\right)}+\frac{J_{c}}{\sigma}\left(1-e^{-\left(\frac{\Delta t}{\tau}\right)}\right)
$$

where $\Delta t$ is the time-step of the calculation (one day), $J_{c}$ is the charging current $\left[\mathrm{A} \mathrm{m}^{-2}\right]$ to the plate, $\sigma$ is the total conductivity $\left[\mathrm{S} \mathrm{m}^{-1}\right]$.

Results of the modelling for the three fixed conductivity cases are shown in Figure 3. As the conductivity reduces, the electric fields becomes larger and the associated time constants becomes longer leading to an integrating and smoothing effect. The electric fields reached for the $10^{-18} \Omega^{-1}$ $\mathrm{m}^{-1}$ conductivity are determined by long term average currents (months) even though sudden increases can also occur e.g. due to the storm in April 2010. As initially noted by Bodeau [14] peak fields do not coincide with peak daily fluences due to the integration effects. For a material with such a low conductivity with this shielding level $(0.5 \mathrm{~mm} \mathrm{Al})$ there would clearly always be the threat of a discharge as the electric field is well above the $10^{7} \mathrm{Vm}^{-1}$ for most of the time. A similar conclusion applies to the dielectric with conductivity $10^{-17} \Omega^{-1} \mathrm{~m}^{-1}$. For the $10^{-16} \Omega^{-1} \mathrm{~m}^{-1}$ conductivity the electric field does remain below the nominal discharge threshold for most of the time with occasional exceedances. These results are qualitatively similar to those obtained by Bodeau but these apply to MEO and are derived from direct charging current measurements. Clearly similar models could also be created using the other two SURF plates to explore the effects of greater shielding levels.

\section{Modelling Radiation INDUCED CONDUCtivity EFFECTS}

The above results are almost certainly pessimistic as most dielectrics exhibit some degree of radiation induced conductivity (RIC). The RIC can be calculated from the 


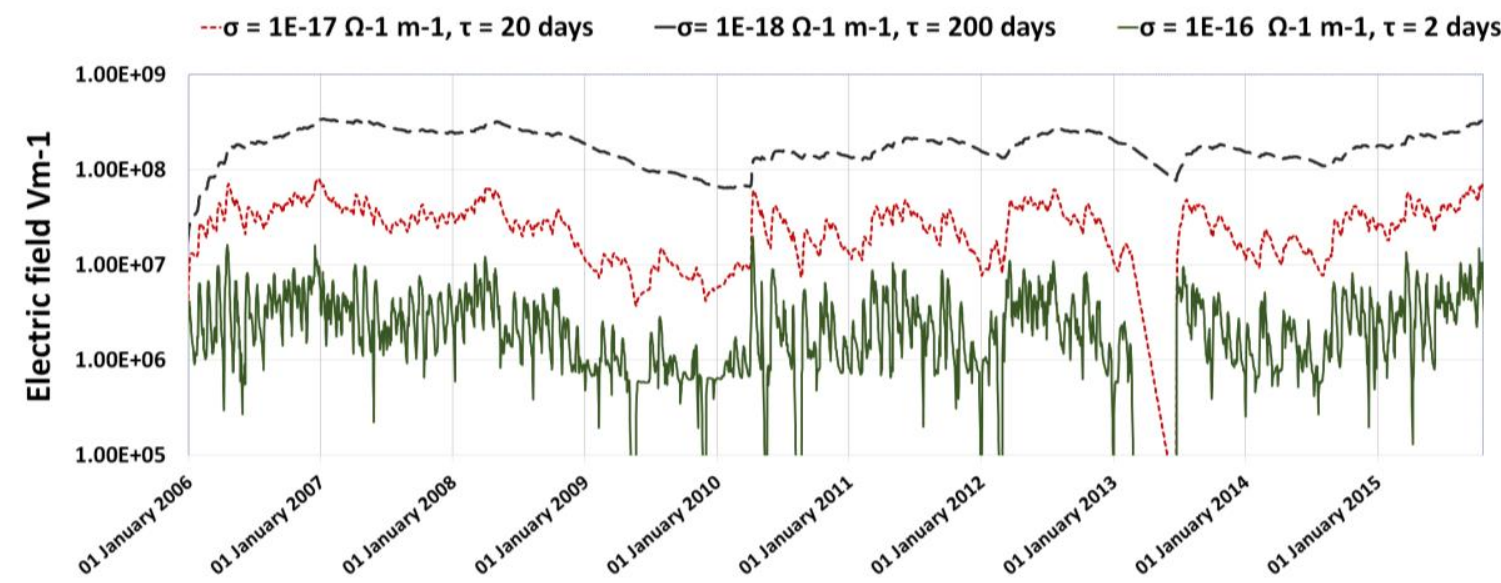

Figure 3 Results from modelling the electric field developed in a $0.5 \mathrm{~mm}$ Al-eq thick dielectric with a $0.5 \mathrm{~mm}$ Al shield over a 10 year period in GPS-like medium Earth orbit. Three different bulk conductivities are modeled which correspond with the time constants shown. RIC is excluded. Results in early 2013 are distorted due to a data gap.

Fowler equation [18]:

$$
\sigma_{R I C}=k_{p} \dot{D}^{\Delta}
$$

where $k_{\mathrm{p}}$ is the co-efficient of RIC [S m${ }^{-1} \mathrm{rad}^{-1} \mathrm{~s}$ ], $\dot{D}$ is the dose rate $\left[\mathrm{rad} \mathrm{s}^{-1}\right]$ and $\Delta$ is a dimensionless material dependent constant.

To calculate the RIC we need to know the dose rate in the model dielectric. We can gain some information on the dose rate in the model dielectric (corresponding to the top plate) from the current in the plates underneath since these lowerlevel deposited currents must have first passed through the top plate. If we sum the currents in the two lower plates we can use this 'transmitted' current to approximate a dose rate in the top plate. Electrons stopping in the top plate also create RIC but is much less significant for this model than that due to the transmitted electrons since we assume that current can only leak from our model dielectric at its bottom surface which is in contact with the metallized grounded layer: hence the conductivity near the lower face of the dielectric is the critical parameter. The RIC of this bottom interface layer is determined by the transmitted current rather than the deposited current. If the grounding layer were at the top (nearest the source) then it would be more appropriate to calculate RIC using all three currents combined.

To find the dose rate from the transmitted current we use an approximation also used in the DICTAT tool [19] which relates the current density passing through a surface to the dose rate i.e.:

$$
\dot{D}=k_{r} \cdot J_{t}
$$

where:

$J$ is the transmitted current density $\left[\mathrm{A} \mathrm{cm}^{-2}\right]$.

$\dot{D}$ is the dose rate $\left[\mathrm{rad} \mathrm{s}^{-1}\right]$

$k_{r}$ is a constant $\left[\mathrm{rad} \mathrm{s}^{-1} \mathrm{~A}^{-1} \mathrm{~cm}^{2}\right]$.
The RIC parameters $k_{p}$ and $\Delta$ have been measured experimentally for selected materials e.g. [20]. PEEK is one material which is used in an increasing number of real spacecraft applications. Recent results from a very long term (two month) laboratory test of PEEK polymer under very low (space-like) electron fluxes with a realistic electron spectrum [21] have determined the RIC parameters as shown in Table 2. We thus choose to use this material for our first model which is labeled 'Case A'. We note that PEEK actually seems to have a low RIC co-efficient compared to many other materials. Thus to examine the effect of an increased RIC co-efficient we have 'Case B' in which this parameter is simply increased by factor 5 (no other changes made).

TABLE 2

RADIATION INDUCED CONDUCTIVITY PARAMETERS FOR MATERIALS USED IN THE MODELLING

\begin{tabular}{|l|l|l|l|l|}
\hline Parameter & Units & $\begin{array}{l}\text { Case A A } \\
(\text { PEEK) }\end{array}$ & $\begin{array}{l}\text { Case B } \\
\text { (PEEK with } \\
\text { increased RIC) }\end{array}$ & $\begin{array}{l}\text { Case C } \\
\text { (Kapton) }\end{array}$ \\
\hline $\begin{array}{l}\text { Bulk } \\
\text { conductivity* } \\
\left(\sigma_{\mathrm{T}}\right)\end{array}$ & $\mathrm{S} \mathrm{m}^{-1}$ & $1.36 \times 10^{-18}$ & $1.36 \times 10^{-18}$ & $1.0 \times 10^{-18}$ \\
\hline $\begin{array}{l}\text { RIC index } \\
(\Delta)\end{array}$ & - & 1.0 & 1.0 & 0.7 \\
\hline $\begin{array}{l}\text { RIC scale } \\
\text { factor }(\mathrm{k})\end{array}$ & $\begin{array}{l}\mathrm{S} \mathrm{m}^{-1} \\
\mathrm{rad}^{-1} \\
\mathrm{~S}\end{array}$ & $4 \times 10^{-16}$ & $2 \times 10^{-15}$ & $4 \times 10^{-15}$ \\
\hline
\end{tabular}

*At room temperature

In addition a further material, Kapton, is modelled using parameters derived by other experimental methods [1] (i.e. not the long term electron testing): these parameters are listed as Case C.

The results of the electric field modelling with RIC i.e. cases A, B and C are shown in Figure 4 which also includes a case without RIC (bulk conductivity $1 \times 10^{-18} \Omega^{-1} \mathrm{~m}^{-1}$ ) for comparison. Looking at Case A (PEEK) there is some 
reduction in the electric field due to RIC but actually the field remains at very high levels indicating a risk of ESD. If $k_{p}$ is made greater by factor 5 (i.e. Case B) the electric field is further suppressed as expected but still remains above the usual $10^{7} \mathrm{Vm}^{-1}$ 'safety threshold'. In Case C (Kapton) the field is further reduced and remains below the 'safety threshold' throughout: this plot is a little more 'spikey' but the range of electric field values is somewhat constrained. In fact the April 2010 storm event which was very significant in terms of charging current does not show up as a major peak and is similar to many other events in its amplitude. This can be explained by the fact that if the RIC co-efficient is large, an increase in charging current tends to be accompanied by significant increase in RIC (transmitted current tends to follow in a similar, albeit not identical fashion). Thus the net effect is to mitigate the increased charging current and so constraining the field.

The shielding level $(0.5 \mathrm{~mm} \mathrm{Al})$ used in the model is quite low by comparison with that which is likely to be afforded to the interior of electronics boxes (which might typically be $>2 \mathrm{~mm}$ Al-eq). The modelling could be performed with 1.0 $\mathrm{mm}$ Al-eq shielding using existing data in which case just the bottom plate would be used for calculating dose rates and RIC. While many dielectric structures on a spacecraft will see levels of shielding in the range $0.5-1.0 \mathrm{~mm} \mathrm{Al-eq} \mathrm{e.g.} \mathrm{in} \mathrm{the}$ harness, it would be useful in future to deploy SURF instruments with plates located at greater shielding depths ( $>2 \mathrm{~mm}$ Al-eq) to address the risks inside of electronics boxes.

It should be noted that temperature can also have major influence on dielectric dark conductivity (a factor of 10 change over a $10 \mathrm{~K}$ range is possible). We have not included this effect so far in our modelling to avoid confusion but since the functional dependence of conductivity vs temperature is usually fairly straightforward (at least over restricted temperature ranges), it could be introduced in future. The actual temperature profile of a location of interest on the spacecraft could be used.

Given that high electric fields are predicted the question arises of whether SURF experienced any ESD events itself. In fact the exposed elements in SURF are primarily grounded (or quasi-grounded) metal and there is minimal exposure of dielectrics. So far there are no indications of any discharge events occurring, but it should be realized that there is no ESD counter (unlike CRRES for example).

\section{CONCLUSION}

We have developed a new internal charging risk indictor by modelling the internal electric fields within on-board dielectrics by using real charging currents measured in flight. Our method also attempts to include the effect of RIC which is a significant mitigating factor. To carry out the modelling we have used data from the SURF instrument in a GPS-type MEO orbit, a region which is of critical importance for space infrastructure. The results support the conclusion from [14] that long term average charging currents (weeks to months) need to be taken into account when assessing risks from highly insulating materials.

The inclusion of RIC in the model does reduce the electric fields developed as would be expected but it does not necessarily prevent an ESD risk from arising. For example $1 \mathrm{~mm}$ thick PEEK would almost always be at risk of breakdown in a MEO (GPS-like) orbit with $0.5 \mathrm{~mm} \mathrm{Al}$ shielding according to our results. The introduction of RIC seems to have a smoothing effect on the electric fields making them less sensitive to perturbation by space weather storms (e.g. April 2010 event). This seems physically reasonable but we also know that some satellite anomalies do show a correlation with storms and short term (daily) electron fluences e.g. [4] so further work is needed to understand the mechanisms involved. Correlating anomaly data sets with electric field models would be a useful future goal in which

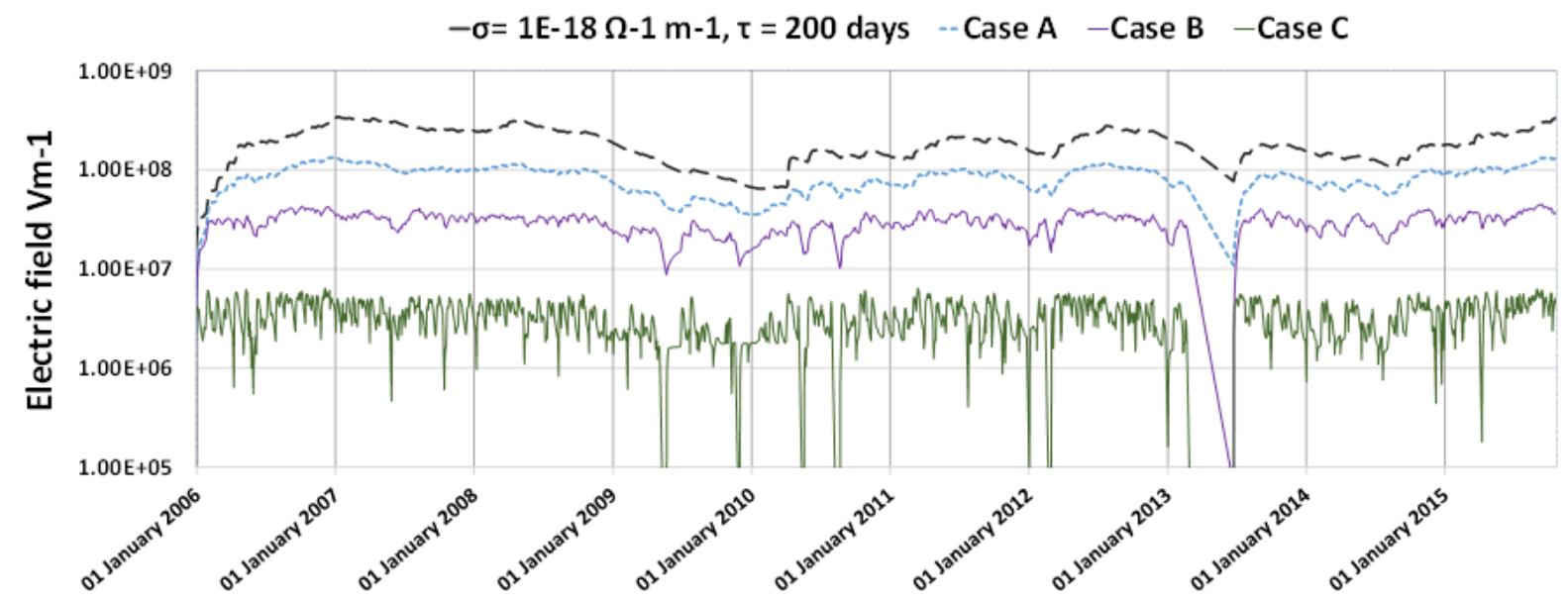

Figure 4 Results of modelling the electric field developed in a $0.5 \mathrm{~mm} \mathrm{Al-eq} \mathrm{thick} \mathrm{dielectric} \mathrm{with} \mathrm{a} 0.5 \mathrm{~mm}$ Al shield over a 10 year period in GPS-like medium Earth orbit including allowance for radiation induced conductivity. Three different bulk conductivities are modeled which correspond with the time constants shown. Results in early 2013 are distorted due to a data gap. 
case we should take account of the fact that if anomalies (i.e. discharges) occur the electric field will 'reset' each time which would have a major influence in electric field timeprofiles. Temperature variations should also be included in future modeling to account for the significant thermal influences on dark conductivity of dielectrics.

Deployment of SURF-like instruments in other orbits would enable similar (possibly real-time) electric field modeling and an immediate priority should be geostationary orbit. Future instruments should contain plates with higher levels of shielding which more closely represent the shielding typically applied to the interiors of electronics boxes ( $>2 \mathrm{~mm}$ Al-eq). We also recommend the development of new flight instruments to measure dielectric internal electric fields and /or ESD events to be flown in combination with SURF-type devices. This would allow much better validation of the modelling results.

\section{ACKNOWLEDGMENT}

We would like to thank SSTL (especially Richard Hebden and Sam Rason) for their support and for supplying us with the on-going data.

\section{REFERENCES}

[1] D. J. Rodgers and K.A. Ryden, "Internal Charging in Space", Proceedings of the $7^{\text {th }}$ Spacecraft Charging Technology Conference, ESA-ESTEC, Noordwijk, The Netherlands, ESA SP-476, p25, 2001.

[2] A. R. Frederickson, Upsets related to spacecraft charging, IEEE TNS, Vol. 43, Issue 3, DOI: 10.1109/23.490891, 1996

[3] R. D. Leach, Failures and anomalies attributed to spacecraft charging, NASA-RP-1375, 1995.

[4] G. L. Wrenn, D. J. Rodgers, and K. A. Ryden, 'A solar cycle of spacecraft anomalies due to internal charging', Annales Geophysicae (2002) 20: 953-956.

[5] www.swpc.noaa.gov

[6] NASA-HDBK-4002A, NASA Technical Handbook: Mitigating InSpace Charging Effects - A Guideline. (03 March 2011)

[7] ECSS-E-ST-20-06C, Spacecraft Charging (2008), 12-15, 1998.

[8] G. L. Wrenn, D. J. Rodgers and P. Buehler, "Modeling the outer belt enhancements of penetrating electrons" J. Spacecraft and Rockets, vol. 37, pp408-415, 2000

[9] D. J. Rodgers. K. A. Hunter and G. L. Wrenn, "The FLUMIC electron environment model", in Proc. $8^{\text {th }}$ Spacecraft Charging Technology Conference, Huntsville, Alabama, NASA/CP-2004-213091, Oct 2004.

[10] A. Hands, K. Ryden, C. Underwood et al 'A New Model of Outer Belt Electrons for Dielectric Internal Charging (MOBE-DIC)', Vo. 62, Issue 6, DOI: 10.1109/TNS.2015.2475134, 2015.

[11] A.R. Frederickson et al, 'Characteristics of Spontaneous Electrical Discharging of Various Insulators in Space Radiations', IEEE TNS, vol. 39. no. 6, Dec 1992.

[12] A.R. Frederickson et al, Radiation induced insulator discharge pulses in the IDM satellite experiment, IEEE TNS, VOL. 38, NO. 6, December 1991.

[13] K. A. Ryden, H. Jolly, A. Frydland and P. Morris, "A Compact electrostatic charging monitor for spacecraft", in Proc. $5^{\text {th }}$ RADECS Conference, Abbaye-de-Fontevraud, Loire, France, Sept 1999.

[14] Bodeau, M. , 'High Energy Electron Climatology that Supports Deep Charging Risk Assessment in GEO', AIAA 2010-1608, Proc. 48th AIAA, Aerospace Sciences, 2010.

[15] T. Mulligan Skov; J. F. Fennell; J. L. Roeder; J. B. Blake; S. G. Claudepierre, 'Internal Charging Hazards in Near-Earth Space During
Solar Cycle 24 Maximum: Van Allen Probes Measurements', IEEE Transactions on Plasma Science, Volume: 43, Issue: 9, DOI: 10.1109/TPS.2015.2468214, 2015.

[16] K. A. Ryden, A.D P. Hands, P. A. Morris, C. S. Dyer, K. A. Ford, B. Taylor, C. I. Underwood, S. Jason, D. Rodgers, G. Mandorlo, G. Gatti, H. D. Evans, E. J. Daly, "Observations of internal charging currents in medium Earth orbit", IEEE Transactions on Plasma Science, Vol. 36, No.5, October 2008.

[17] K. A. Ryden; A. D. P. Hands; C. I. Underwood; D. J. Rodgers, 'Internal Charging Measurements in Medium Earth Orbit Using the SURF Sensor: 2005-2014', IEEE Transactions on Plasma Science, Volume: 43, Issue: 9, DOI: 10.1109/TPS.2015.2416436, 2015.

[18] J. F. Fowler, 'Radiation-induced Conductivity in the Solid State, and Some Applications', Physics in Medicine and Biology, Volume 3, Number 4, DOI, http://dx.doi.org/10.1088/0031-9155/3/4/307 (1959).

[19] D. Rodgers, K. A. Ryden, G. L. Wrenn et al, 'Engineering Tools for Internal Charging', ESA Contract Final Report, DERA/CIS(CIS2)/7/36/2/4 Issue 1.0, (1999).

[20] T. Paulmier; B. Dirassen; M. Arnaout; D. Payan; N. Balcon, 'RadiationInduced Conductivity of Space Used Polymers Under High Energy Electron Irradiation', IEEE Transactions on Plasma Science, Volume: 43, Issue: 9, DOI: 10.1109/TPS.2015.2452943, 2015.

[21] A. Hands and K. Ryden, 'Experimental Measurement of Low Intensity Long Duration Internal Charging Behaviour', (submitted to this issue of TPS).

[22] D. Rodgers, K. A. Ryden, G. L. Wrenn et al, 'Engineering Tools for Internal Charging', ESA Contract Final Report, DERA/CIS/CIS2/7/CR000277, Issue 1.0, (2000).

Keith A. Ryden received a BSc(Hons) degree in Electrical and Electronic Engineering from the University of Bath, England, in 1986 and an MSc in Satellite Engineering from the University of Surrey, Guildford, England, in 1988.

From 1986 to 2000 he worked for the Ministry of Defence (MoD) at the Royal Aerospace Establishment (RAE) Farnborough, England, carrying out research to support the UK Skynet military satellite constellation, focusing especially on radiation and plasma environments and their effects on electronic systems. As part of this work he led the development of the STRV1a/b radiation effects research satellites which were launched in 1994. He then moved on to work at QinetiQ (2000-2013) where he was Leader for Space Environment and Effects and worked on numerous MoD, European Space Agency (ESA) and industrial projects. He was appointed to be a QinetiQ Technical Fellow in 2007.

Keith Ryden currently holds the position of Reader in Space Engineering at the University of Surrey Space Centre where his research interests cover space and atmospheric radiation environments and its effects on technology, especially single event effects, ionizing dose and electrostatic charging. He is Principal Investigator for a number of radiation monitors deployed in space and in the upper atmosphere. He was a coauthor of the Royal Academy of Engineering report 'Extreme Space Weather: Impacts on Engineered Systems and Infrastructure' (2013) and is a member of the UK Space Environment Impact Expert Group (SEIEG) which advises government. Mr Ryden is a Chartered Engineer (CEng) and a 
Fellow of the Institution of Engineering and Technology

(FIET) 